\title{
Desenvolvimento pós-embrionário de Anteos menippe (Hübner) (Lepidoptera, Pieridae) em Cassia ferruginea Shrad. (Caesalpinaceae), em laboratório
}

\author{
Flávia de Souza Born ${ }^{1} \&$ Iracilda Maria de Moura Lima²
}

\begin{abstract}
'Departamento de Fitotecnia e Fitossanidade/Zootecnia, Universidade Federal de Alagoas. flaviaborn@gmail.com
${ }^{2}$ Departamento de Zoologia, Universidade Federal de Alagoas. Praça Afrânio Jorge, s/n, Prado, 57.010-020 Maceió-AL, Brasil. iralima20@gmail.com
\end{abstract}

\begin{abstract}
Development stadia of Anteos menippe (Hübner) (Lepidoptera, Pieridae) on Cassia ferruginea Shrad. (Caesalpinaceae), in laboratory. In order to determine the duration of the post-embryonic development and its instars, eggs of Anteos menippe were collected from Cassia ferruginea leaves and were taken into the laboratory to register the dates of ecdysis. The post-embryonic development was (in days average \pm standard error) $25.7 \pm 0.4$, the larval period $15.9 \pm 0.3$ represented $61.7 \%$ of the development and had five instars: $\mathrm{L} 1=2.2 \pm 0.1, \mathrm{~L} 2=1.7 \pm 0.2, \mathrm{~L} 3=2.0 \pm 0.1$, $\mathrm{L} 4=3.4 \pm 0.1$ and $\mathrm{L} 5=6.4 \pm 0.3$. This last instar had an active phase $(5.4 \pm 0.3)$ and a prepupal phase ( 1 day long). The pupal period was $9.9 \pm 0.2$. The inactivity period (prepupa + pupa) $10.9 \pm 0.2$ days represented $42.2 \%$ of the postembryonic development, and the active phase $57.8 \%$. The egg viability was $91.6 \%$ and the survival, starting from neonate larvae, was $59.8 \%$.
\end{abstract}

KEYWORDS. Coliadinae; ontogeny; host-plant; sex ratio.

RESUMO. Desenvolvimento pós-embrionário de Anteos menippe (Hübner) (Lepidoptera, Pieridae) em Cassia ferruginea Shrad. (Caesalpinaceae), em laboratório. Com o objetivo de estabelecer a duração do desenvolvimento pós-embrionário e dos instares, foram coletados ovos de Anteos menippe em folhas de Cassia ferruginea e levados ao laboratório para o registro das datas das ecdises. O desenvolvimento pós-embrionário durou (média \pm erro-padrão) $25,7 \pm 0,4$ dias. $\mathrm{O}$ período larval $(15,9 \pm 0,3$ dias $)$ representou $61,7 \%$ do desenvolvimento e contou com cinco instares: $\mathrm{L} 1=2,2 \pm 0,1 ; \mathrm{L} 2=$ $1,7 \pm 0,2 ; \mathrm{L} 3=2,0 \pm 0,1 ; \mathrm{L} 4=3,4 \pm 0,1$ e $\mathrm{L} 5=6,4 \pm 0,3$. Este último ínstar contou com uma fase ativa $(5,4 \pm 0,3$ dias $)$ e prepupa (1 dia). O período pupal foi de $9,9 \pm 0,2$ dias. O período de inatividade (prepupa + pupa) foi de 10,9 $\pm 0,2$ dias representando $42,2 \%$ do desenvolvimento pós-embrionário, sendo o período ativo (larval ativo) 57,8\% do desenvolvimento. A viabilidade de ovos foi de $91,6 \%$ e a sobrevivência, a partir do número de lagartas neonatas foi de $59,8 \%$.

PALAVRAS-CHAVE. Coliadinae; ontogenia; planta-hospedeira; razão-sexual.

Segundo Mielke \& Casagrande (1997), “o estudo da riqueza das espécies de uma determinada área é de extrema importância, não só para o conhecimento histórico, como também para o conhecimento global da biodiversidade". Aliado a esse princípio, o conhecimento de aspectos biológicos de espécies urbanas de insetos é de grande importância para a avaliação da qualidade ambiental e para o estabelecimento de procedimentos que visam à preservação de ambientes.

Cassia ferruginea Shrad. (Caesalpinaceae) é muito utilizada na arborização urbana. Dentre os fitófagos a ela associados, encontram-se as lagartas de Anteos menippe (Hübner, 1818) (Lepidoptera, Pieridae) cujos adultos são muito comuns nos jardins públicos e de residências tanto em áreas urbanas quanto rurais.

Como plantas-hospedeiras das lagartas de A. menippe, Otero (1986, 1990) registrou Cassia siamea Lam. (Caesalpinaceae), utilizada na arborização urbana no Brasil em cidades como Belém, Recife, Maceió, Salvador e Rio de Janeiro e Figueiredo (2000a, b) a indica para a região Sudeste do Brasil. Silva et al. (1968) apresentam Acacia sp. e espécies de crucíferas (Brassicaceae), sem, no entanto, especificá-las. Silva (1983) registra a ocorrência de $A$. menippe para o Estado de Alagoas.

$\mathrm{Na}$ família Pieridae, apenas Ascia monuste orseis (Latreille, 1819) (Pieridae, Pierini) (lagarta-da-couve ou curuquerê-dacouve) é incluída como praga no Brasil, por causar sérios danos a crucíferas (Otero 1986).

Este trabalho visou estabelecer a duração, em dias, de cada um dos instares do desenvolvimento pós-embrionário de $A$. menippe em $C$. ferruginea, determinar a percentagem de sobrevivência durante este desenvolvimento e calcular a razão sexual da espécie.

\section{MATERIAL E MÉTODOS}

O trabalho foi realizado no Laboratório de Entomologia do Departamento de Zoologia da Universidade Federal de Alagoas, em 2000, em condições ambientes, com registro de temperatura e umidade relativa do ar através de termohigrômetro digital.

Os ovos foram coletados em plantas de C. ferruginea (Fig.1), recolhidos no próprio folíolo da oviposição. No laboratório, cada ovo foi isolado juntamente com o fragmento 

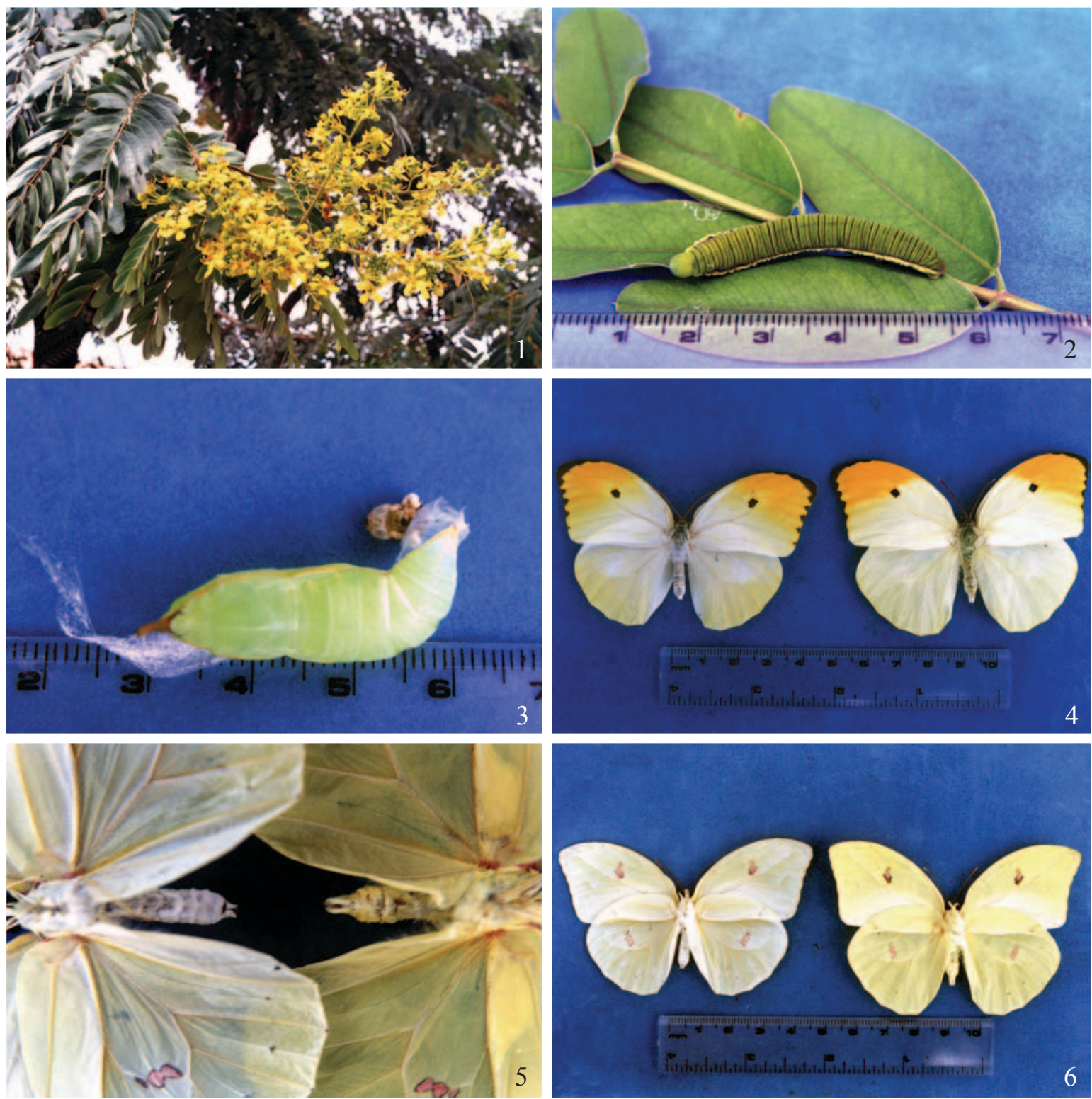

Figs. 1-6. 1, aspecto geral de ramo de Cassia ferruginea Schard. (Caesalpinaceae); 2-6, Anteos menippe (Hübner, 1818) (Lepidoptera, Pieridae): 2, lagarta no quinto instar; 3 , pupa; 4, adultos em vista dorsal (fêmea à esquerda); 5 , vista ventral da terminália (fêmea à esquerda); 6 , vista ventral dos adultos (fêmea à esquerda).

da folha onde estava aderido, colocado sobre papel absorvente umedecido com água em tubo branco de filme fotográfico coberto com tecido fino. Após a eclosão das larvas, era feita limpeza diária substituindo as folhas, sendo as observações anotadas em planilhas de acompanhamento.

Uma vez que as lagartas geralmente consumiam toda a exúvia do tórax e abdome, apenas as cápsulas cefálicas foram guardadas como provas das ecdises.

As lagartas no terceiro instar eram transferidas para gaiolas de plástico cobertas com tecido fino com liga de borracha contendo grande quantidade de folhas menos tenras, com papel absorvente umedecido na base dos ramos para manter a turgidez. As gaiolas foram confeccionadas com garrafas plásticas de refrigerante de 2,0 L tendo como base tampas plásticas. As lagartas empuparam especialmente na parte superior dos ramos, onde permaneceram até a data da emergência dos adultos.

Os adultos e suas exúvias pupais foram incorporados à Coleção Entomológica Prof. José Cordeiro da Silva, Departamento de Zoologia, Universidade Federal de Alagoas.

A razão sexual foi calculada com a divisão do número de fêmeas pelo total de indivíduos (machos + fêmeas) (SilveiraNeto et al. 1976).

Foram calculadas as medidas de tendência central (Média, Moda e Mediana) e as de dispersão (Amplitude, incluindo valores mínimo e máximo, Coeficiente de Variação e Intervalo de Confiança da Média) com um nível de confiança de 95\%, indicado para experimentos biológicos dessa natureza (Pagano \& Gauvreau 2004). Também foram determinados a assimetria e o grau de achatamento da curva, para confirmar o coeficiente 


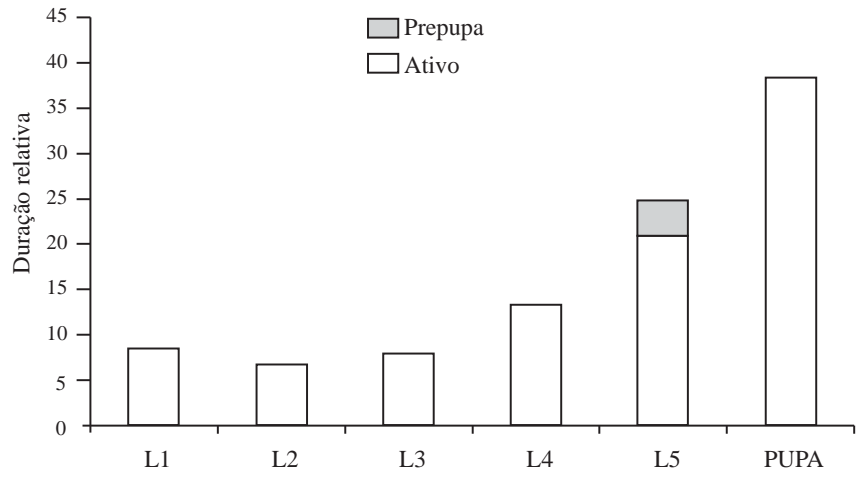

Fig. 7 - Duração relativa dos instares do desenvolvimento pósembrionário de Anteos menippe (Hübner, 1818) (Lepidoptera, Pieridae) alimentando-se de Cassia ferruginea Schard. (Caesalpinaceae), em laboratório $\left(25,5^{\circ} \mathrm{C}\right.$ e $70 \%$ UR).

de variação, o qual foi enquadrado em quatro classes: I, homogêneo $(<10 \%)$; II, relativamente homogêneo (10-20\%); III, relativamente heterogêneo (20-30\%); e, IV, heterogêneo $(>30 \%)$.

\section{RESULTADOS}

O período pós-embrionário, antes de atingir a fase adulta, está representado por cinco instares larvais e o pupal (Figs. 2, 3, Tabela I).

Primeiro instar. A duração absoluta (Tabela I) teve uma variação de 2 a 4 dias (amplitude de 2 dias), sendo a média de 2,2 dias com Intervalo de Confiança da Média de 2,0 a 2,4 dias. O valor médio representou uma duração relativa (Tabela II, Fig. 7) de $14 \%$ do período larval. Os dados obtidos podem ser considerados relativamente heterogêneos com coeficiente de variação de 23,4\%. A distribuição apresentou moda de 2 dias (igual à mediana), indicando uma assimetria positiva e forte. $\mathrm{O}$ grau de achatamento foi de 7,3630, indicando que a distribuição é leptocúrtica.
Segundo instar. A duração absoluta (Tabela I) teve uma variação de 1 a 4 dias (amplitude de 3 dias), sendo a média de 1,7 dias com Intervalo de Confiança da Média de 1,4 a 2,1 dias. $\mathrm{O}$ valor médio representou uma duração relativa (Tabela II, Fig. 7) de $11 \%$ do período larval. O coeficiente de variação foi $46,6 \%$, a distribuição foi bimodal ( 1 e 2 dias) e mediana de 2 dias, indicando uma assimetria negativa e fraca. O coeficiente de curtose foi de 3,7721 , caracterizando a distribuição como leptocúrtica.

Terceiro instar. A duração absoluta (Tabela I) teve uma variação de 1 a 3 dias (amplitude de 2 dias), sendo a média de 2,0 dias com Intervalo de Confiança da Média de 1,9 a 2,2 dias. $\mathrm{O}$ valor médio representou uma duração relativa (Tabela II, Fig. 7) de 12,9\% do período larval. Os dados obtidos foram relativamente homogêneos com coeficiente de variação de $18,0 \%$. A distribuição apresentou moda e mediana igual a 2 dias, indicando uma assimetria positiva e fraca. O coeficiente de curtose foi de 7,5466, caracterizando a distribuição como leptocúrtica.

Quarto instar. A duração absoluta (Tabela I) teve uma variação de 3 a 5 dias (amplitude de 2 dias), sendo a média de 3,4 dias com Intervalo de Confiança da Média de 3,1 a 3,7 dias. O valor médio representou uma duração relativa (Tabela II, Fig. 7) de 21,6\% do período larval. Os dados foram homogêneos com coeficiente de variação de 19,3\%. A distribuição apresentou moda e mediana igual a 3 dias, indicando uma assimetria positiva e fraca. $\mathrm{O}$ coeficiente de curtose foi de 3,2825, caracterizando a distribuição como leptocúrtica.

Quinto instar. O quinto instar apresentou duas fases distintas denominadas de fase ativa e prepupa. A fase ativa apresentou uma duração absoluta (Tabela I) variando de 3 a 8 dias (amplitude de 5 dias), sendo a média de 5,4 dias com Intervalo de Confiança da Média de 4,9 a 5,9 dias. O valor médio representou uma duração relativa (Tabela II, Fig. 7) de $34 \%$ do período larval. Os dados obtidos podem ser considerados heterogêneos uma vez que o coeficiente de variação foi 22,2\%. A distribuição apresentou moda e mediana

Tabela I. Estatísticas descritivas da duração em dias, das fases do desenvolvimento pós-embrionário de Anteos menippe (Hübner, 1818) (Lepidoptera, Pieridae) alimentando-se de folhas de Cassia ferruginea Schard. (Caesalpinaceae), em laboratório $\left(25,5^{\circ} \mathrm{C}\right.$ e $70 \%$ UR).

\begin{tabular}{|c|c|c|c|c|c|c|}
\hline \multirow{2}{*}{\multicolumn{2}{|c|}{ Fase }} & \multicolumn{3}{|c|}{ Duração (dias) } & \multirow[b]{2}{*}{$\mathrm{CV}^{*}(\%)$} & \multirow[b]{2}{*}{ Assimetria } \\
\hline & & Média \pm SM & Moda/Mediana & $\begin{array}{l}\text { Amplitude Total } \\
\text { (Mínimo-Máximo) }\end{array}$ & & \\
\hline Larva 1 & & $2,2 \pm 0,11$ & $2 / 2$ & $2(2-4)$ & 23,4 & $+1,2731$ \\
\hline Larva 2 & & $1,7 \pm 0,2$ & 1 e $2 / 2$ & $3(1-4)$ & 46,6 & $-0,9629$ \\
\hline Larva 3 & & $2,0 \pm 0,1$ & $2 / 2$ & $2(1-3)$ & 18,0 & $+0,3273$ \\
\hline Larva 4 & & $3,4 \pm 0,1$ & $3 / 3$ & $2(3-5)$ & 19,3 & $+1,9475$ \\
\hline \multirow[t]{3}{*}{ Larva 5} & - Ativa & $5,4 \pm 0,3$ & $6 / 6$ & $5(3-8)$ & 22,2 & $-1,5299$ \\
\hline & - Prepupa & 1,0 & $1 / 1$ & $0(1-1)$ & 0,0 & 0,0000 \\
\hline & - Total & $6,4 \pm 0,3$ & $7 / 7$ & $5(4-9)$ & 18,7 & $-1,5299$ \\
\hline \multicolumn{2}{|c|}{ Larval Ativo } & $14,9 \pm 0,3$ & $14 / 14$ & $5(13-18)$ & 9,6 & $+1,8330$ \\
\hline \multicolumn{2}{|c|}{ Larval Total } & $15,9 \pm 0,3$ & $15 / 15$ & $5(14-19)$ & 9,0 & $+1,8330$ \\
\hline \multicolumn{2}{|c|}{ Pupal } & $9,9 \pm 0,2$ & $10 / 10$ & $5(7-12)$ & 11,1 & $-0,3546$ \\
\hline \multicolumn{2}{|c|}{ Período de inat.** } & $10,9 \pm 0,2$ & $11 / 11$ & $7(8-13)$ & 10,1 & $-1,7007$ \\
\hline \multicolumn{2}{|c|}{ TOTAL } & $25,7 \pm 0,4$ & $26 / 26$ & $7(23-30)$ & 6,4 & $-0,4706$ \\
\hline
\end{tabular}

* CV - Coeficiente de variação

** Período de inat. - Período de inatividade (= período prepupal + período pupal) 
Tabela II. Duração absoluta (média) em dias e duração relativa à fase larval e à duração do desenvolvimento pós-embrionário de Anteos menippe (Hübner, 1818) (Lepidoptera, Pieridae) alimentando-se de folhas de Cassia ferruginea Schard. (Caesalpinaceae), em laboratório $\left(25,5^{\circ} \mathrm{C}\right.$ e $70 \%$ UR $)$.

\begin{tabular}{|c|c|c|c|}
\hline \multirow{3}{*}{ Fase } & \multicolumn{3}{|c|}{ Duração } \\
\hline & \multirow{2}{*}{$\begin{array}{l}\text { Absoluta } \\
\text {-Média- } \\
\text { (dias) }\end{array}$} & \multicolumn{2}{|c|}{ Relativa (\%) } \\
\hline & & Á Fase Larval & Ao Período Total \\
\hline Larva 1 & 2,2 & 14,0 & 8,6 \\
\hline Larva 2 & 1,7 & 11,0 & 6,8 \\
\hline Larva 3 & 2,0 & 12,9 & 7,9 \\
\hline Larva 4 & 3,4 & 21,6 & 13,3 \\
\hline \multicolumn{4}{|l|}{ Larva 5} \\
\hline - Ativa & 5,4 & 34,0 & 20,9 \\
\hline - Prepupa & 1,0 & 6,3 & 3,9 \\
\hline - Total & 6,4 & 40,3 & 24,8 \\
\hline Larval Ativo & 14,9 & 93,7 & 57,8 \\
\hline Larval Total & 15,9 & - & 61,7 \\
\hline Pupal & 9,9 & - & 38,3 \\
\hline TOTAL & 25,7 & - & - \\
\hline Período de inat.** & 10,9 & - & 42,2 \\
\hline
\end{tabular}

igual a 6 dias, indicando uma assimetria negativa e fraca. $\mathrm{O}$ coeficiente de curtose foi de 2,6159, caracterizando a distribuição como platicúrtica.

O período prepupal não apresentou variação (Tabelas I, II, Fig. 7), uma vez que em todos os espécimens a duração foi de um dia. A duração total do quinto instar apresentou uma duração absoluta (Tabela I) variando de 4 a 9 dias (amplitude de 5 dias), sendo a média de 6,4 dias com Intervalo de Confiança da Média de 5,9 a 6,9 dias. O valor médio representou uma duração relativa (Tabela II, Fig. 7) de 40,3\% do período larval.

Os dados obtidos podem ser considerados relativamente homogêneos uma vez que o coeficiente de variação foi $18,7 \%$. A distribuição apresentou moda e mediana igual a 7 dias, indicando uma assimetria negativa e fraca. O coeficiente de curtose foi de 2,6113, igual ao da fase ativa deste estádio, caracterizando a distribuição como platicúrtica.

Período larval ativo. A duração absoluta (Tabela I) teve uma variação de 13 a 18 dias (amplitude de 5 dias), sendo a média de 14,9 dias com Intervalo de Confiança da Média 14,3 a 15,5 dias. O valor médio representou uma duração relativa (Tabela II) de 93,7\% do período larval. Os dados obtidos apresentam um coeficiente de variação de $9,6 \%$. A distribuição apresentou moda e mediana igual a 14 dias, indicando uma assimetria positiva e fraca. O coeficiente de curtose foi de 2,3632, caracterizando a distribuição como platicúrtica.

Período larval total. A duração absoluta (Tabela I) teve uma variação de 14 a 19 dias (amplitude de 5 dias), sendo a média 15,9 dias com Intervalo de Confiança da Média de 15,3 a 16,5 dias. O valor médio representou uma duração relativa (Tabela II) de $61,7 \%$ do período pós-embrionário total $(25,7$ dias). Os dados obtidos podem ser considerados homogêneos uma vez que o coeficiente de variação foi $9 \%$. A distribuição apresentou moda e mediana igual a 15 dias, indicando uma assimetria positiva e fraca. O coeficiente de curtose foi de
2,3632, igual ao período larval ativo, caracterizando a distribuição como platicúrtica.

Em A. monuste orseis foram observados valores próximos aos de A. menippe. Fancelli \& Vendramim (1992) registraram períodos médios de duração do período larval entre 13,02 a 14,74 dias, em diferentes cultivares de Brassica oleraceae L. var. Acephala DC $\left(25 \pm 2{ }^{\circ} \mathrm{C}\right.$ e $80 \pm 10 \%$ UR); Bortoli et al. (1983) obtiveram entre 11,4 e 16,2 (dias). Valores inferiores, entre 10,2 e $10,8 \operatorname{dias}\left(28 \pm 5^{\circ} \mathrm{C}, 75 \% \mathrm{UR}\right)$ foram obtidos por Catta-Preta \& Zuculoto (2003).

Período pupal. A duração absoluta (Tabela I) teve uma variação de 7 a 12 dias (amplitude de 5 dias), sendo a média de 9,9 dias com Intervalo de Confiança da Média de 9,4 a 10,4 dias. O valor médio representou uma duração relativa (Tabela II, Fig. 7) de 38,3\% do período pós-embrionário total $(25,7$ dias). Os dados obtidos apresentam coeficiente de variação de $11,1 \%$. A distribuição apresentou moda e mediana igual a 10 dias, indicando uma assimetria negativa e fraca. $O$ coeficiente de curtose foi de 3,6278, caracterizando a distribuição como leptocúrtica. O período pupal (Fig.3), com média de 9,9 dias, representa mais do que a soma da duração dos quatro primeiros ínstares larvais (9,4 dias).

Duração total do desenvolvimento pós-embrionário. A duração absoluta (Tabela I) teve uma variação de 23 a 30 dias (amplitude de 7 dias), sendo a média de 25,7 dias com Intervalo de Confiança da Média de 25,0 a 26,5 dias. O coeficiente de variação foi $6,4 \%$. A distribuição apresentou moda e mediana igual a 26 dias, indicando uma assimetria negativa e fraca. $\mathrm{O}$ coeficiente de curtose foi de 3,5769, caracterizando a distribuição como leptocúrtica.

Período de inatividade dentro do desenvolvimento pósembrionário. A duração absoluta (Tabela I) teve uma variação de 8 a 13 dias (amplitude de 5 dias), sendo a média de 10,9 dias com Intervalo de Confiança da Média de 10,4 a 11,3 dias. O valor médio representou uma duração relativa (Tabela II) de $42,2 \%$ do período pós-embrionário total ( 25,7 dias). Os dados

Tabela III. Viabilidade de ovos e sobrevivência, em cada um dos períodos do desenvolvimento pós-embrionário de Anteos menippe (Hübner, 1818) (Lepidoptera, Pieridae) alimentando-se de folhas de Cassia ferruginea Schard. (Caesalpinaceae), em laboratório $\left(25,5^{\circ} \mathrm{C}\right.$ e $70 \%$ UR).

\begin{tabular}{lcccc}
\hline \multirow{2}{*}{$\begin{array}{l}\text { Período do } \\
\text { Desenvolvimento }\end{array}$} & $\begin{array}{c}\text { Unidades de Prova } \\
\text { (número) }\end{array}$ & \multicolumn{2}{c}{$\begin{array}{c}\text { Sobrevivência } \\
(\%)\end{array}$} \\
\cline { 2 - 5 } & Inicial & Final & No Estádio & Acumulada \\
\hline Embrionário & & & & \\
(Viabilidade) & 95 & 87 & 91,6 & - \\
L1 & 87 & 67 & 77,0 & 77,0 \\
L2 & 67 & 63 & 94,0 & 72,4 \\
L3 & 63 & 60 & 95,2 & 69,0 \\
L4 & 60 & 60 & 100,0 & 69,0 \\
L5 & 60 & 57 & 95,0 & 65,5 \\
Larval total & 87 & 57 & 65,5 & 65,5 \\
Pupal: & 57 & 52 & 91,2 & 59,8 \\
$\quad$ Falha na emergência & 57 & 55 & 96,5 & 63,2 \\
$\quad$ Morte no estádio & 55 & 52 & 94,6 & 59,8 \\
\hline \multicolumn{1}{c}{ Total } & 57 & 52 & 91,2 & 59,8 \\
\hline
\end{tabular}


obtidos podem ser considerados relativamente homogêneos uma vez que o coeficiente de variação foi $10,1 \%$. A distribuição apresentou moda e mediana igual a 11 dias, indicando uma assimetria negativa e fraca. O coeficiente de curtose foi de 2,1014, caracterizando a distribuição como platicúrtica.

Razão sexual. Em vista dorsal, machos e fêmeas são muito semelhantes (Fig. 4); em vista ventral os machos apresentam coloração amarela mais viva (Fig. 6). No entanto, a maior evidência de dimorfismo para a realização da sexagem é a terminália (Fig. 5).

Dos 23 espécimens do experimento sobre desenvolvimento pós-embrionário que atingiram a fase adulta, 13 foram fêmeas e 10 machos, o que dá uma proporção sexual de 1: 0,77 machos, representando uma razão sexual de 0,57.

Viabilidade de ovos e sobrevivência ao longo do desenvolvimento pós-embrionário. $\mathrm{O}$ acompanhamento das perdas de unidades de prova ao longo do bioensaio, pode ser observado na Tabela III. Dos 95 ovos coletados no campo, com data de postura não conhecida, de oito não eclodiram larvas, o que representa uma viabilidade de $91,6 \%$. A partir daí, foi analisada a sobrevivência analisando-se o registro das mortes.

Em relação à sobrevivência das larvas ao longo do desenvolvimento, ao se comparar o valor obtido para $A$. menippe com os de outros Lepidoptera, verifica-se uma perda significativa de efetivos ao longo do período larval, perfazendo uma sobrevivência no total desse estágio de 59,8\% inferior ao obtido para espécies como $A$. monuste orseis $96,8 \%$ (Pereira $e t$ al. 2003). No entanto, a sobrevivência pode variar em função do alimento como registraram Bortoli et al. (1983) para $A$. monuste orseis, em diferentes cultivares de Brassica oleraceae L. (Brassicaceae), com valores entre 56,6 e 85\%.

Dentro do período pupal a sobrevivência foi de $91,2 \%$, semelhante à obtida para $A$. monuste orseis por Fancelli \& Vendramim (1992) (96,5\%) e à registrada por Bortoli et al. (1983) (entre 80,8 e 92,9\%), mostrando-se superior à observada por Pereira et al. (2003) (61,5\%).

Agradecimentos. À Coordenação de Aperfeiçoamento de Pessoal de Nível Superior (CAPES) pela concessão de bolsa de mestrado à primeira autora, ao Prof. Dr. Olaf Hermann Hendrik Mielke pela confirmação da espécie Anteos menippe (Hübner, 1818) (Lepidoptera, Pieridae), à bióloga Rosângela Lyra Lemos pela identificação de Cassia ferruginea Shrad. (Caesalpinaceae) e aos avaliadores anônimos pelas valiosas e pertinentes sugestões.

\section{REFERÊNCIAS}

Barros, W. R. S. \& I. M. M. Lima. 2004. Desenvolvimento pré-imaginal de Eueides isabela dianasa (Hübner, 1806) (Lepidoptera: Nymphalidae: Heliconiinae) alimentando-se de folhas de Pasiflora edulis L. (Passifloraceae). Revista Brasileira de Entomologia 48: 69-75.

Bortoli, S. A.; D. A. Banzato \& A. C. Forner. 1983. Aspectos biológicos comparados de Ascia monuste orseis (Latreille, 1819) (LepidopteraPiralidae) em três cultivares de couve (Brassica oleraceae v. acephala). Anais da Sociedade Entomológica do Brasil 12: 284-289.

Catta-Preta, P. D. \& F. S. Zuculoto. 2003. Oviposition behavior and performance aspects of Ascia monuste orseis (Godart, 1919) (Lepidoptera, Pieridae) on kale (Brassica oleraceae var. acephala). Revista Brasileira de Entomologia 47: 169-174.

Fancelli, M. \& J. D. Vendramim. 1992. Aspectos biológicos de Ascia monuste orseis (Godart, 1819) (Lepidoptera, Pieridae) em cultivares de couve. Anais da Sociedade Entomológica do Brasil 21: $357-366$.

Figueiredo, O. S. 2000a. Criação de borboletas. Viçosa, CPT, 78 p. (Pequenas Criações; n. 281).

Figueiredo, O. S. 2000b. Manual de criação de borboletas: mensageiras da paz. Campinas, Osmar Salles de Figueiredo, 78 p.

Mielke, O. H. H. \& M. M. Casagrande. 1997. Papilionoidea e Hesperioidea (Lepidoptera) do Parque Estadual do Morro do Diabo, Teodoro Sampaio, São Paulo, Brasil. Revista Brasileira de Zoologia 14: 967-1001.

Otero, L. S. 1986. Borboletas: livro do naturalista. Rio de Janeiro, FAE - Ministério da Educação, 112 p.

Otero, L. S. 1990. Butterflies: beauty and behavior of brazilian species. Rio de Janeiro, Marigo Comunicação Visual, 127 p.

Pagano, M. \& K. Gauvreau. 2004. Princípios de Bioestatística. São Paulo, Pioneira Thomson Learning, 506 p.

Pereira, T.; A. Pasini \& É. D. M. Oliveira. 2003. Biologia e preferência alimentar de Ascia monuste orseis (Latreille) (Lepidoptera: Pieridae) na planta invasora Raphanus raphanistrum L. Neotropical Entomology 32: 725-727.

Santos, G. P.; J. C. Zanuncio; A. P Alves \& T. V. Zanuncio. 1993. Biologia de Fulgurodes sartinaria Guenée (Lepidoptera, Geometridae) em Pinus patula. Revista Brasileira de Zoologia 10: $321-325$.

Silva, A. G. D’a.; C. R. Gonçalves; D. M. Galvão; A. J. L. Gonçalves; J. Gomes; M. do N. Silva \& L. de Simoni. 1968. Quarto Catálogo dos insetos que vivem nas plantas do Brasil seus parasitos e predadores: insetos, hospedeiros e inimigos naturais. Parte 2: Tomo 1. Rio de Janeiro, Ministério da Agricultura, 622 p.

Silva, J. C. da. 1983. Contribuição ao estudo dos insetos do Estado de Alagoas - I. Maceió, Scientia ad sapientiam 11: 10-15.

Silveira-Neto, S.; O. Nakano; D. Barbin \& N. A. Villa-Nova. 1976. Manual de ecologia dos insetos. Piracicaba, Agronômica Ceres, $419 \mathrm{p}$.

Recebido em 15.III.2003; aceito em 15.IV.2005 